\title{
SUR LES REPRÉSENTATIONS MIXTES DES GROUPES DE LIE RÉSOLUBLES EXPONENTIELS
}

\author{
A. Baklouti, A. Ghorbel et H. Hamrouni
}

\begin{abstract}
Let $G$ be an exponential solvable Lie group, $H$ and $A$ two closed connected subgroups of $G$ and $\sigma$ a unitary and irreducible representation of $H$. We prove the orbital spectrum formula of the Up-Down representation $\rho(G, H, A, \sigma)=\operatorname{Ind}_{H}^{G} \sigma_{\mid A}$. When $G$ is nilpotent, the multiplicities of such representation turns out to be uniformly infinite or finite and bounded. A necessary and sufficient condition for the finiteness of the multiplicities is given. The same results are obtained when $G$ is exponential solvable Lie group, $H$ and $A$ are invariant.
\end{abstract}

\section{Introduction}

Soit $G$ un groupe de Lie résoluble exponentiel d'algèbre de Lie $\mathfrak{g}$ et $H \subset G$ un sous-groupe fermé connexe. Soient $\pi$ et $\sigma$ deux représentations unitaires et irréductibles de $G$ et de $H$ respectivement. H. Fujiwara a déterminé des formules explicites de désintégration en irréductibles des représentations $\operatorname{Ind}_{H}^{G} \sigma$ et $\pi_{\mid H}$ en termes de spectre et de multiplicités de représentations (voir $[\mathbf{8}]$ et $[\mathbf{9}]$ ). On se demande si on peut obtenir des formules analogues pour des représentations qui font intervenir à la fois des représentations induites et des restrictions de représentations. C'est en fait ce qu'on appelle dans ce travail des représentations mixtes ou de type mixte. Plus précisément, ce sont des représentations de la forme $\rho(G, H, A, \sigma)=\operatorname{Ind}_{H}^{G} \sigma_{\mid A}$. L'objet de ce travail est l'étude de ce type de représentations. Comme motivation, l'étude de telles représentations porte un grand intérêt sachant que la décomposition des représentations mixtes et leur entrelacement nous fournit de nouvelles désintégrations lisses de $L^{2}(G)$ pour les groupes exponentiels (voir [1]).

2000 Mathematics Subject Classification. 22 E 27.

Mots-clés. Groupe exponentiel, représentation unitaire, orbite, désintégration, polarisation, ensemble semi-algébrique. 
Une autre motivation vient originalement de la théorie des actions ergodiques comme l'explique R. L. Lipsman dans [12]. Ce dernier auteur étudie le spectre et les multiplicités d'un type particulier de représentations mixtes pour des groupes de Lie nilpotents connexes et simplement connexes. C'est la représentation

$$
\rho(G, H, A)=\operatorname{Ind}_{H}^{G} 1_{\mid A}
$$

qui était l'objet de l'étude en supposant que $H$ est un sous-groupe fermé de $G$ et $A$ un sous-groupe abélien de $G$. Plus précisément, l'auteur a déterminé un spectre concret et une formule de multiplicités explicite en utilisant la méthode des orbites. Notre premier objectif dans cet article est d'écrire des formules de désintégration des représentations mixtes pour tous les groupes de Lie résolubles exponentiels sans restrictions sur les sous-groupes $H$ et $A$ et sur la représentation $\sigma \in \hat{H}$. Un autre objectif de ce travail est de déterminer des conditions nécessaires et suffisantes pour lesquelles les multiplicités des représentations intervenant dans les composantes isotypiques de $\rho(G, H, A, \sigma)$ sont finies presque partout. Pour acquérir de telles conditions, il faut déjà que la représentation $\tau(\sigma)=\operatorname{Ind}_{H}^{G} \sigma$ soit à multiplicités finies et que pour presque tout $\pi \in \operatorname{spectre}(\tau(\sigma))$ la représentation $\pi_{\mid A}$ soit encore à multiplicités finies. Ces dernières conditions ne sont généralement pas suffisantes pour assurer la finitude des multiplicités de la représentation $\rho(G, H, A, \sigma)$.

Quand $G$ est nilpotent, nous démontrons que les multiplicités de la représentation mixte $\rho(G, H, A, \chi)$ sont uniformément infinies ou uniformément finies et bornées. Nous exhibons aussi dans ce cas une condition nécessaire et suffisante pour la finitude de ces multiplicités. Ici, $\chi$ désigne un caractère unitaire de $H$. Cette condition porte manifestement sur les sous-groupes $H$ et $A$ et leurs actions sur certains espaces en faisant intervenir des ensembles semi-algébriques. Le fait de travailler en utilisant un caractère unitaire ne représente aucune perte de généralités dans ce cas. En effet, toutes les représentations unitaires et irréductibles sont monomiales et le résultat est déductible pour une représentation quelconque $\sigma$ de $H$. Quand le groupe $G$ est exponentiel et $A$ s'avère une polarisation $G$-invariante pour certains éléments génériques, nous montrons que les multiplicités de la représentation $\rho(G, H, A, \sigma)$ coïncident avec celle de $\tau(\sigma)$ et sont uniformes, donc ne dépendent pas de $A$. Lorsque les sous-groupes $H$ et $A$ sont invariants (et $G$ est exponentiel), nous montrons que la représentation mixte $\rho(G, H, A, \chi)$ est sans multiplicités ou à multiplicités uniformément égales à l'infini. Nous donnons alors des conditions nécessaires et suffisantes pour la finitude dans ce cas. 
L'étude de telles représentations pourrait être faite plus généralement sur des groupes localement compacts. Mackey a étudié la désintégration de telles représentations quand $A$ et $H$ sont régulièrement liés (voir [17]). D'autre part J. Ludwig prouve dans le Lemme 4.2 de [16] que si $H$ et $A$ sont des sous-groupes normaux d'un groupe localement compact $G$ et $A \subset H$, alors le support de la représentation mixte $\rho(G, H, A, \sigma)$ est de la forme $\left(\sigma_{\mid A}\right)^{g}, g \in G$ où $\left(\sigma_{\mid A}\right)^{g}(a)=\sigma\left(g \cdot a \cdot g^{-1}\right), a \in A$.

\section{Généralités}

Soit $G$ un groupe de Lie résoluble exponentiel d'algèbre de Lie $\mathfrak{g}$, c'est à dire un groupe de Lie réel tel que l'application exponentielle:

$$
\exp : \mathfrak{g} \longrightarrow G
$$

soit un difféomorphisme. Soit $\mathfrak{g}^{\star}$ l'espace dual de $\mathfrak{g}$. Le groupe $G$ agit sur $\mathfrak{g}^{\star}$ par la représentation coadjointe. Pour tout $f$ dans $\mathfrak{g}^{\star}$, on définit la forme bilinéaire alternée $B_{f}$ sur $\mathfrak{g} \times \mathfrak{g}$ par $B_{f}(X, Y)=f([X, Y])$. Si a est une sous-algèbre de $\mathfrak{g}$, on note $\mathfrak{a}(f)=\left\{X \in \mathfrak{g} / B_{f}(X, \mathfrak{a})=0\right\}$ et $\mathfrak{a}^{\perp}=\left\{l \in \mathfrak{g}^{\star}: l_{\mid \mathfrak{a}}=0\right\}$ où $l_{\mid \mathfrak{a}}$ désigne la restriction de $l$ à $\mathfrak{a}$. Si $\mathfrak{a} \subset$ $\mathfrak{a}(f)$, on dit que $\mathfrak{a}$ est une sous-algèbre isotrope pour $B_{f}$. On note aussi $S(f, \mathfrak{g})$ l'ensemble des sous-algèbres isotropes pour $B_{f}$. Etant donné un élément $\mathfrak{h}$ de $S(f, \mathfrak{g})$, on note $\chi_{f}$ le caractère unitaire de $H=\exp (\mathfrak{h})$ défini par

$$
\chi_{f}(\exp (Y))=e^{i f(Y)}, \quad Y \in \mathfrak{h} .
$$

Ce caractère induit une représentation unitaire $\tau=\tau(f)=\operatorname{Ind}_{H}^{G} \chi_{f}$ de $G$. Soit $\sigma$ une représentation de $H$ associée à l'orbite $\Omega_{\sigma}^{H}$, qui agit sur un espace de Hilbert $\mathcal{H}_{\sigma}$ et construisons la représentation $\tau(\sigma)=\operatorname{Ind}_{H}^{G} \sigma$. Nous allons préciser dans ce qui suit la façon avec laquelle on définit la représentation $\tau(\sigma)$. Soient $d g$ une mesure de Haar à gauche sur $G$ et $\Delta_{G}$ la fonction module de $G$ de sorte que l'on a:

$$
\int_{G} F\left(g x^{-1}\right) d g=\Delta_{G}(x) \int_{G} F(g) d g \quad(x \in G)
$$

pour toute fonction $F$ appartenant à l'espace $K(G)$ des fonctions continues sur $G$ à support compact. Il est bien connu que

$$
\Delta_{G}(x)=|\operatorname{det} \operatorname{Ad} x|^{-1} \quad(x \in G) .
$$

On note $\Delta_{H, G}$ le caractère de $H$ à valeurs dans $\mathbf{R}_{+}$défini par

$$
\Delta_{H, G}(h)=\frac{\Delta_{H}(h)}{\Delta_{G}(h)} .
$$


On a, pour $X$ dans $\mathfrak{h}$,

$$
\Delta_{H, G}(\exp X)=\exp \left(\operatorname{Tr}_{\operatorname{ag}} \operatorname{glh}_{\mathfrak{h}} X\right) .
$$

Soit $K(G, H)$ l'espace des fonctions numériques $F$ continues sur $G$, à support compact modulo $H$ et qui vérifient:

$$
F(g h)=\Delta_{H, G}(h) F(g) \quad(g \in G, h \in H) .
$$

$G$ agit sur cet espace par translation à gauche. On sait ([5]) que, à un scalaire multiplicatif près, il existe une et une seule forme linéaire positive $G$-invariante sur $K(G, H)$. On la note $\nu_{G, H}$ et on l'écrit sous la forme d'une intégrale:

$$
\nu_{G, H}(F)=\oint_{G / H} F(g) d \nu(g) .
$$

Si $\Delta_{H}=\Delta_{G}$ sur $H, \nu_{G, H}$ (c'est par exemple le cas quand $H$ est un sous-groupe normal de $G$ ) est une mesure $G$-invariante sur l'espace homogène $G / H$.

On note $K(\sigma, \mathfrak{h}, G)$ l'espace des fonctions $F$ continues sur $G$ à valeurs dans $\mathcal{H}_{\sigma}$ et qui vérifient:

$$
F(g h)=\Delta_{H, G}^{1 / 2}(h) \sigma(h)^{-1} F(g) \quad(g \in G, h \in H)
$$

et dont le support est compact modulo $H$. Si $F$ appartient à $K(\sigma, \mathfrak{h}, G)$, la fonction $g \mapsto\|F(g)\|_{\sigma}^{2}$ appartient à $K(G, H)$ et l'on pose:

$$
\|F\|^{2}=\oint_{G / H}\|F(g)\|_{\sigma}^{2} d \nu(g) .
$$

La représentation induite $\tau(\sigma)=\operatorname{Ind}_{H}^{G} \sigma$ de $G$ se réalise par translation à gauche dans l'espace de Hilbert $\mathcal{H}_{\tau}(\sigma)$ complété de $K(\sigma, \mathfrak{h}, G,\|\cdot\|)$ muni de la norme définie ci-dessus.

Soit $M(f, \mathfrak{g})$ l'ensemble des éléments de $S(f, \mathfrak{g})$ de dimension maximale et $I(f, \mathfrak{g})$ le sous-ensemble de $S(f, \mathfrak{g})$ formé des sous-algèbres $\mathfrak{h}$ telles que $\tau(f)$ soit irréductible. Alors il est bien connu qu'un élément $\mathfrak{h}$ de $S(f, \mathfrak{g})$ est dans $I(f, \mathfrak{g})$ si et seulement si $\mathfrak{h}$ vérifie la condition de Pukanszky, à savoir que $H \cdot f=f+\mathfrak{h}^{\perp}, \mathfrak{h}^{\perp}$ désignant l'orthogonal de $\mathfrak{h}$ dans $\mathfrak{g}^{\star}$; on dira dans ce cas que $\mathfrak{h}$ est une polarisation de Pukanszky. Cette dernière condition est vraie pour tout élément de $M(f, \mathfrak{g})$ lorsque $G$ est nilpotent. 
Lorsque $\mathfrak{h}$ ne vérifie pas la condition de Pukanszky, alors la représentation $\tau(f)$ n'est plus irréductible. On la décompose en une intégrale hilbertienne de représentations unitaires irréductibles. C'est le sousespace affine $\Gamma_{f}=\Gamma_{\tau(f)}=f+\mathfrak{h}^{\perp}$ qui porte la mesure de désintégration de $\tau(f)$. Notons $\hat{G}$ le dual unitaire de $G$, soit $\Theta: \mathfrak{g}^{\star} \rightarrow \hat{G}$ l'application de Kirillov-Bernat, le dual unitaire de $G, \hat{G}$ est paramétré par l'espace des orbites coadjointes $\mathfrak{g}^{\star} / G$ au moyen de la bijection induite $\bar{\Theta}: \mathfrak{g}^{\star} / G \rightarrow \hat{G}$ (cf. [5]). Pour $\pi \in \hat{G}$ on note $\Omega_{\pi}^{G}=\Theta^{-1}(\pi)$ l'orbite associée. Soit $q: \mathfrak{g}^{\star} \rightarrow \mathfrak{h}^{\star}$ et $p: \mathfrak{g}^{\star} \rightarrow \mathfrak{a}^{\star}$ les projections canoniques de $\mathfrak{h}$ et $\mathfrak{a}$. Il existe sur la sous-variété $q^{-1}\left(\Omega_{\sigma}^{H}\right)$ de $\mathfrak{g}^{\star}$ une mesure $\tilde{\mu}$ bien déterminée par la mesure canonique sur $\Omega_{\sigma}^{H}$ et par la mesure de Lebesgue sur l'orthogonal de $\mathfrak{h}$ dans $\mathfrak{g}^{\star}$. On prend une mesure finie sur $\mathfrak{g}^{\star}$ équivalente à $\tilde{\mu}$ qu'on note aussi $\tilde{\mu}$. Nous prenons l'image $\mu$ de $\tilde{\mu}$ par l'application de KirillovBernat.

D'après H. Fujiwara (cf. [8]), la représentation $\tau(\sigma)$ satisfait la formule orbitale du spectre; à savoir que:

$$
\tau(\sigma) \simeq \int_{G \cdot q^{-1}\left(\Omega_{\sigma}^{H}\right) / G}^{\oplus} n_{\phi}^{\sigma} \pi_{\phi} d \nu_{G, H}^{\sigma}(\phi),
$$

où $\nu_{G, H}^{\sigma}$ est l'image par l'application $q^{-1}\left(\Omega_{\sigma}^{H}\right) \rightarrow G \cdot q^{-1}\left(\Omega_{\sigma}^{H}\right) / G$ de la mesure naturelle sur $q^{-1}\left(\Omega_{\sigma}^{H}\right) \subset \mathfrak{g}^{\star}$ et $n_{\phi}^{\sigma}$ est le nombre des $H$-orbites contenues dans $q^{-1}\left(\Omega_{\sigma}^{H}\right) \cap G \cdot \phi$ (voir aussi [2], [7] et [14]).

Gardons nos hypothèses, $G=\exp (\mathfrak{g})$ un groupe de Lie résoluble exponentiel, $H=\exp (\mathfrak{h})$ et $A=\exp (\mathfrak{a})$ deux sous-groupes de $G$. Considérons maintenant une représentation unitaire et irréductible $\pi$ associée à une orbite coadjointe $\Omega_{\pi}=\Omega_{\pi}^{G} \subset \mathfrak{g}^{\star}$ d'une forme linéaire $f$. Soit $\pi_{\mid A}$ la restriction de $\pi$ à $A$ et $\mu_{\pi}$ une mesure finie sur $\Omega_{\pi}$ équivalente à la mesure $G$-invariante. Alors d'après H. Fujiwara (cf. $[\mathbf{9}]$ ), la restriction $\pi_{\mid A}$ satisfait la formule orbitale du spectre, c'est à dire:

$$
\pi_{\mid A}=\int_{p\left(\Omega_{\pi}\right) / A}^{\oplus} n_{\pi}^{\psi} \sigma_{\psi} d \mu_{G, A}^{\pi}(\psi)
$$

où $n_{\pi}^{\psi}=\#\left[\left(\Omega_{\pi} \cap p^{-1}(A \cdot \psi)\right)\right] / A$ et $\mu_{G, A}^{\pi}$ est la mesure image de la mesure canonique sur $\Omega_{\pi}$ par l'application $\Omega_{\pi} \longrightarrow p\left(\Omega_{\pi}\right) / A$ (voir aussi [3] et $[\mathbf{1 3}])$. 


\subsection{Représentations mixtes.}

Définition 2.1. Soit $G$ un groupe de Lie, $H$ et $A$ deux sous-groupes de $G$ et $\sigma$ une représentation de $H$. On appelle représentation mixte ou de type mixte la représentation

$$
\rho(G, H, A, \sigma)=\operatorname{Ind}_{H}^{G} \sigma_{\mid A} .
$$

Lorsqu'on remplace la représentation unitaire $\sigma$ de $H$ par un caractère unitaire $\chi_{f}$, la représentation sera tout simplement notée par $\rho(G, H, A, f)$. Alors, il est clair que $\rho(G, H, G, \sigma)=\tau(\sigma)$ et $\rho(G, G, A, \sigma)=\sigma_{\mid A}$. D'autre part, si le produit $A H$ est un sous-groupe fermé de $G$, alors le théorème du sous-groupe de Mackey nous permet d'affirmer que

$$
\rho(A H, H, A, \sigma) \simeq \rho\left(A, A \cap H, A, \sigma_{\mid A \cap H}\right) \simeq \operatorname{Ind}_{A \cap H}^{A}\left(\sigma_{\mid A \cap H}\right) .
$$

Si $G$ est résoluble exponentiel et si $\sigma$ est une représentation unitaire irréductible de $H$, alors $\sigma$ est une représentation monomiale, c'est à dire induite d'un caractère unitaire. Notons $\sigma \simeq \operatorname{Ind}_{B}^{H} \chi_{l}$ où $l \in \mathfrak{g}^{\star}$, $B=\exp (\mathfrak{b})$ et $\mathfrak{b} \in I\left(l_{\mid \mathfrak{h}}, \mathfrak{h}\right)$. Alors il est immédiat que

$$
\rho(G, H, A, \sigma) \simeq \rho(G, B, A, l) .
$$

Ainsi l'étude de la représentation mixte (5) quand $\sigma \in \hat{H}$ pourra être effectuée sur des représentations mixtes de type $\rho(G, H, A, f)$. C'est ce qu'on trouve d'ailleurs dans beaucoup de références. Dans ce texte, certains de nos résultats seront exprimés pour des représentations de type mixte d'ordre général comme dans (5). La relation (6) s'avère parfois trés utile pour transférer certains résultats établis sur des représentations mixtes de type $\rho(G, H, A, f)$ à des représentations mixtes générales.

Quand $A=H$, la représentation $\rho(G, H, H, \sigma)$ sera notée tout simplement $\rho(G, H, \sigma)$.

\section{Désintégration des représentations mixtes}

Dans cette section, nous allons écrire la désintégration de la représentation $\rho(G, H, A, \sigma)$ tout en donnant une description explicite de l'espace de désintégration, des mesures et des multiplicités des représentations unitaires irréductibles qui apparaissent dans cette décomposition. Le résultat principal de cette section est: 
Théorème 3.1. Soit $G$ un groupe de Lie résoluble exponentiel, $H$ et $A$ deux sous-groupes fermés connexes de $G$ et $\sigma$ une représentation unitaire et irréductible de $H$. Alors la représentation mixte $\rho(G, H, A, \sigma)$ satisfait la formule orbitale du spectre à savoir:

$$
\rho(G, H, A, \sigma)=\int_{\left[p\left(G \cdot q^{-1}\left(\Omega_{\sigma}^{H}\right)\right)\right] / A}^{\oplus} m_{\sigma}(\psi) \rho_{\psi} d \mu_{G, H, A}^{\sigma}(\psi),
$$

où $\mu_{G, H, A}^{\sigma}$ est la mesure image de $(d g \times \tilde{\mu})$ sur $G \times q^{-1}\left(\Omega_{\sigma}^{H}\right)$ au moyen des applications

$$
G \times q^{-1}\left(\Omega_{\sigma}^{H}\right) \rightarrow G \cdot q^{-1}\left(\Omega_{\sigma}^{H}\right) \rightarrow p\left(G \cdot q^{-1}\left(\Omega_{\sigma}^{H}\right)\right) \rightarrow\left[p\left(G \cdot q^{-1}\left(\Omega_{\sigma}^{H}\right)\right)\right] / A
$$

et

$$
m_{\sigma}(\psi)=\sum_{\Omega \in\left[G \cdot q^{-1}\left(\Omega_{\sigma}^{H}\right) \cap G \cdot p^{-1}(\psi)\right] / G} n_{\Omega}^{\sigma} n_{\Omega}^{\psi}
$$

ò̀ $n_{\Omega}^{\sigma}=\#\left[\left(\Omega \cap q^{-1}\left(\Omega_{\sigma}^{H}\right)\right)\right] / H$ et $n_{\Omega}^{\psi}=\#\left[\left(\Omega \cap p^{-1}(A \cdot \psi)\right)\right] / A$.

Remarque. Quand $G$ est nilpotent, $A$ est abélien et $\sigma=1_{H}$, il a été prouvé dans $[\mathbf{1 2}$, Théorème 3.1] que la représentation $\rho(G, H, A)$ définie par la relation (1) satisfait la formule orbitale du spectre, à savoir

$$
\rho(G, H, A)=\int_{p\left(G \cdot h^{\perp}\right)}^{\oplus} n_{\psi} \chi_{\psi} d \psi \quad \text { et } \quad n_{\psi}=\sum_{\Omega \in\left[G \cdot \mathfrak{h}^{\perp} \cap G \cdot p^{-1}(\psi)\right] / G} n_{\Omega}^{\sigma} n_{\Omega}^{\psi} .
$$

Preuve du Théorème 3.1: La preuve de ce théorème repose sur les désintégrations de l'induite unitaire $\tau(\sigma)$ et des restrictions $\pi_{\phi \mid A}$, où $\pi_{\phi}$ est la représentation lieé à la forme linéaire $\phi \in q^{-1}\left(\Omega_{\sigma}^{H}\right)$. D'aprés la formule (3) nous avons

$$
\tau(\sigma)=\int_{G \cdot q^{-1}\left(\Omega_{\sigma}^{H}\right) / G}^{\oplus} n_{\phi}^{\sigma} \pi_{\phi} d \nu_{G, H}^{\sigma}(\phi)
$$

Il en résulte:

$$
\begin{aligned}
\rho(G, H, A, \sigma) & =\left(\int_{G \cdot q^{-1}\left(\Omega_{\sigma}^{H}\right) / G}^{\oplus} n_{\phi}^{\sigma} \pi_{\phi} d \nu_{G, H}^{\sigma}(\phi)\right)_{\mid A} \\
& =\int_{G \cdot q^{-1}\left(\Omega_{\sigma}^{H}\right) / G}^{\oplus} n_{\phi}^{\sigma} \pi_{\phi} d \nu_{G, H}^{\sigma}(\phi) .
\end{aligned}
$$

D'autre part nous avons d'après la formule (4):

$$
\pi_{\phi \mid A}=\int_{p(G \cdot \phi) / A}^{\oplus} n_{\phi}^{\psi} \gamma_{\psi} d \mu_{G, A}^{\pi_{\phi}}(\psi) .
$$


Ainsi:

$$
\begin{aligned}
\rho(G, H, A, \sigma) & =\int_{G \cdot q^{-1}\left(\Omega_{\sigma}^{H}\right) / G}^{\oplus} n_{\phi}^{\sigma} \int_{p(G \cdot \phi) / A}^{\oplus} n_{\phi}^{\psi} \gamma_{\psi} d \mu_{G, A}^{\pi_{\phi}}(\psi) d \nu_{G, H}^{\sigma}(\phi) \\
& =\int_{G \cdot q^{-1}\left(\Omega_{\sigma}^{H}\right) / G}^{\oplus} \int_{p(G \cdot \phi) / A}^{\oplus} n_{\phi}^{\sigma} n_{\phi}^{\psi} \gamma_{\psi} d \mu_{G, A}^{\pi_{\phi}}(\psi) d \nu_{G, H}^{\sigma}(\phi) \\
& =\int_{S}^{\oplus} m(\psi, \phi) \gamma_{\psi} d \mu_{G, A}^{\pi_{\phi}}(\psi) \otimes d \nu_{G, H}^{\sigma}(\phi)
\end{aligned}
$$

où $S=\bigcup_{G \cdot \phi \in\left[G \cdot q^{-1}\left(\Omega_{\sigma}^{H}\right)\right] / G} p(G \cdot \phi) / A$.

Remarquons tout d'abord que la projection $p$ est $\operatorname{Ad}^{*}(A)$-équivariante c-à-d $p \circ \operatorname{Ad}^{*}(a)=\operatorname{Ad}^{*}(a) \circ p$. Comme $G \cdot q^{-1}\left(\Omega_{\sigma}^{H}\right)$ est $G$-invariant, $p\left(G \cdot q^{-1}\left(\Omega_{\sigma}^{H}\right)\right)$ est $A$-invariant. Ainsi

$$
S=\left[p\left(G \cdot q^{-1}\left(\Omega_{\sigma}^{H}\right)\right)\right] / A .
$$

Notons $\tilde{\mu}_{G, H, A}^{\sigma}$ la mesure sur $p\left(G \cdot q^{-1}\left(\Omega_{\sigma}^{H}\right)\right)$ obtenue par image de $(d g \times \tilde{\mu})$ sur $G \times q^{-1}\left(\Omega_{\sigma}^{H}\right)$ au moyen des applications $G \times q^{-1}\left(\Omega_{\sigma}^{H}\right) \rightarrow$ $G \cdot q^{-1}\left(\Omega_{\sigma}^{H}\right) \rightarrow p\left(G \cdot q^{-1}\left(\Omega_{\sigma}^{H}\right)\right)$. Quitte à transporter la mesure $\tilde{\mu}_{G, H, A}^{\sigma}$ sur $p\left(G \cdot q^{-1}\left(\Omega_{\sigma}^{H}\right)\right)$ par l'application $p\left(G \cdot q^{-1}\left(\Omega_{\sigma}^{H}\right)\right) \rightarrow p\left(G \cdot q^{-1}\left(\Omega_{\sigma}^{H}\right)\right) / A$, nous obtenons la mesure $\mu_{G, H, A}^{\sigma}$ sur $\left[p\left(G \cdot q^{-1}\left(\Omega_{\sigma}^{H}\right)\right)\right] / A$ décrite dans le théorème. D'autre part d'aprés les formules (3), (4) la mesure $\nu_{G, H}^{\sigma}$ est l'image de $(d g \times \tilde{\mu})$ par l' application $G \times q^{-1}\left(\Omega_{\sigma}^{H}\right) \rightarrow G \cdot q^{-1}\left(\Omega_{\sigma}^{H}\right)$ et pour $G \cdot \phi \in\left(G \cdot q^{-1}\left(\Omega_{\sigma}^{H}\right)\right) / G$ la mesure $\mu_{G, A}^{\pi_{\phi}}$ est l'image de la mesure canonique sur $G \cdot \phi$ par l'application $G \cdot \phi \rightarrow p(G \cdot \phi) / A$. Comme la classe de la mesure canonique sur l'orbite $G \cdot \phi$ est l'image de la mesure de Haar $d g$ par l'application $G \rightarrow G \cdot \phi$, alors $\mu_{G, A}^{\pi_{\phi}}$ est l'image de $d g$ par l'application $G \rightarrow G \cdot \phi \rightarrow p(G \cdot \phi) / A$. Ainsi moyennant l'égalité (9) on peut voir que, lorsque $G \cdot \phi$ varie dans $\left(G \cdot q^{-1}\left(\Omega_{\sigma}^{H}\right)\right) / G$, le produit tensoriel des mesures $\mu_{G, A}^{\pi_{\phi}}$ et $\nu_{G, H}^{\sigma}$ nous donne également la mesure $\mu_{G, H, A}^{\sigma}$ sur $\left[p\left(G \cdot q^{-1}\left(\Omega_{\sigma}^{H}\right)\right)\right] / A$. Toutes ces remarques et la formule (8) nous permettent d'affirmer que

$$
\rho(G, H, A, \sigma)=\int_{p\left(G \cdot q^{-1}\left(\Omega_{\sigma}^{H}\right)\right) / A}^{\oplus} m(\psi) \rho_{\psi} d \mu_{G, H, A}^{\sigma}(\psi)
$$

pour certaine fonction multiplicités $m(\psi)$ que nous allons prouver qu'elle coincide avec la fonction donnée dans (7). Pour $\psi \in \mathfrak{a}^{\star}$, on pose

$$
\mathcal{M}_{\psi}(\sigma)=\left\{\phi \in G \cdot q^{-1}\left(\Omega_{\sigma}^{H}\right) ; G \cdot \phi \text { rencontre } p^{-1}\{A \cdot \psi\}\right\} .
$$


Comme $p^{-1}\{A \cdot \psi\}=A \cdot p^{-1}\{\psi\}$, il est clair que $\mathcal{M}_{\psi}(\sigma)$ est $G$-invariant et que $\mathcal{M}_{\psi}(\sigma)=G \cdot q^{-1}\left(\Omega_{\sigma}^{H}\right) \cap G \cdot p^{-1}(\psi)$. Ainsi l'ensemble $\mathcal{M}_{\psi}(\sigma)$ ne dépend que de l'orbite $A \cdot \psi$.

On déduit alors la fonction multiplicités

$$
m(\psi)=\sum_{G \cdot \phi \in\left[\mathcal{M}_{\psi}(\sigma)\right] / G} n_{G \cdot \phi}^{\sigma} n_{G \cdot \phi}^{\psi}=\sum_{\Omega \in\left[\mathcal{M}_{\psi}(\sigma)\right] / G} n_{\Omega}^{\sigma} n_{\Omega}^{\psi} .
$$

D'aprés la définition de la fonction de multiplicités $n_{\Omega}^{\psi}$, on peut voir que la fonction $m$ est constante sur chaque $A$-orbite. Ainsi on déduit la formule de désintègration décrite dans le théorème.

Comme résultat immédiat du Théorème 3.1, nous déduisons la désintégration centrale en représentations unitaires irréductibles de la représentation mixte $\rho(G, H, A, \sigma)$. Soit $\mathcal{M}^{\prime}{ }_{\psi}(\sigma)$, l'ouvert dense de $\mathcal{M}_{\psi}(\sigma)$ formé par les éléments dont la $G$-orbite est de dimension maximale. Quand $\sigma=\chi_{f}$, on note $\mathcal{M}_{\psi}(\sigma)=\mathcal{M}_{\psi}$ et $\mathcal{M}^{\prime}{ }_{\psi}(\sigma)=\mathcal{M}^{\prime}{ }_{\psi}$.

Corollaire 3.2. Soit $G$ un groupe de Lie résoluble exponentiel, $H$ et $A$ deux sous-groupes fermés connexes de $G$ et $\sigma$ une représentation unitaire et irréductible de $H$. Soit $\beta$ la mesure image de la mesure $\mu_{G, H, A}^{\sigma}$ sur $\left[p\left(G \cdot q^{-1}\left(\Omega_{\sigma}^{H}\right)\right)\right] / A$ par l'application de Kirillov-Bernat $K_{A}: \mathfrak{a}^{\star} / A \rightarrow \hat{A}$. Alors

$$
\rho(G, H, A, \sigma) \simeq \int_{\hat{A}}^{\oplus} m(\gamma) \gamma d \beta(\gamma)
$$

Une représentation $\gamma=\gamma_{\psi}, \psi \in \mathfrak{a}^{\star}$ intervient dans la décomposition (10) si et seulement si $\psi \in p\left(G \cdot q^{-1}\left(\Omega_{\sigma}^{H}\right)\right)$. Les multiplicités $m\left(\gamma_{\psi}\right)$ de $\rho(G, H, A, \sigma)$ qui sont données comme dans (7) sont finies $\beta$-presque partout si et seulement si $\tau(\sigma), \pi_{\phi \mid A}$ sont à multiplicités finies pour presque tout $\phi \in q^{-1}\left(\Omega_{\sigma}^{H}\right)$ et $\left[\mathcal{M}^{\prime}{ }_{\psi}(\sigma)\right] / G$ est à cardinal fini pour presque tout $\psi \in p\left(G \cdot q^{-1}\left(\Omega_{\sigma}^{H}\right)\right)$.

\section{Multiplicités des représentations mixtes}

Soit $W$ une variété, on dit qu'une propriété $\left\{P_{w}, w \in W\right\}$ est vraie génériquement sur $W$, si elle est vraie en tout point de $W$ sauf sur un ensemble négligeable relativement à une classe de mesure donnée sur $W$.

Nous supposons toujours que $G$ est un groupe de Lie résoluble exponentiel, $H$ et $A$ sont deux sous-groupes fermés connexes de $G$. Il est bien connu que lorsque $G$ est complètement résoluble, les multiplicités des représentations $\tau(\sigma)$ pour $\sigma \in \hat{H}$ et $\pi_{\mid A}$ sont uniformément infinies 
ou uniformément finies et bornées (voir [15]). La condition de la finitude des multiplicités de la représentation $\tau(\sigma)$ est donnée par l'égalité

$$
\operatorname{dim} G \cdot \phi=2 \operatorname{dim} H \cdot \phi-\operatorname{dim} \Omega_{\sigma}^{H}
$$

pour $\phi$ générique dans $q^{-1}\left(\Omega_{\sigma}^{H}\right)$ (voir [14]).

De la même façon, les multiplicités de la restriction $\pi_{\mid A}$ sont uniformément finies quand

$$
\operatorname{dim} \Omega_{\pi}=2 \operatorname{dim} A \cdot \phi-\operatorname{dim} A \cdot p(\phi),
$$

pour $\phi$ générique dans $\Omega_{\pi}$ (voir [13]). Sinon, ces multiplicités sont uniformément égales à l'infini. Le même phénomène est obtenu quand $G$ est résoluble exponentiel et les sous-groupes $H$ et $A$ sont normaux. Nous allons chercher dans cette section des conditions nécessaires et suffisantes pour la finitude des multiplicités des représentations mixtes dans le cas des groupes nilpotents. Nous montrons que de telles multiplicités sont uniformément infinies ou uniformément finies et bornées. Pour aboutir à de tels résultats, on utilise des techniques émanant de la géométrie algébrique.

Nous allons rappeller quelques propriétés des ensembles semi-algébriques. Ce matériel est assez standart. Nous envoyons le lecteur à [4], [6] et [18] pour plus de détails. Un sous-ensemble $V$ de $\mathbf{R}^{n}$ est dit semi-algébrique, s'il est de la forme

$$
V=\left\{x \in \mathbf{R}^{n}: P_{1}(x)=\cdots=P_{s}(x)=0, Q_{1}(x)>0, \ldots, Q_{l}(x)>0\right\}
$$

où les $P_{i}, i=1, \ldots, s$ et les $Q_{j}, j=1, \ldots, l$ sont des fonctions polynomiales sur $\mathbf{R}^{n}$. Un ensemble de $\mathbf{R}^{n}$ sera dit algébrique s'il est l'ensemble des zéros communs d'une famille finie de fonctions polynomiales sur $\mathbf{R}^{n}$.

Chaque ensemble semi-algébrique $V$ de $\mathbf{R}^{n}$ admet une partition $\left\{A_{i}\right\}_{i=1}^{m}$ vérifiant:

1) chaque $A_{i}$ est une sous-variété connexe, localement fermée de $\mathbf{R}^{n}$,

2) si $\overline{A_{i}} \cap A_{j} \neq \emptyset$, alors $A_{j} \subset \overline{A_{i}}$ et $\operatorname{dim} A_{j}<\operatorname{dim} A_{i}$,

3) chaque $A_{i}$ est un ensemble semi-algébrique.

Une telle partition est dite une stratification de $V$. On appelle dimension de $V$ l'entier

$$
d=\max _{1 \leq i \leq m} \operatorname{dim} A_{i} .
$$

Cette définition est indépendante du choix de la stratification $\left\{A_{i}\right\}_{i=1}^{m}$.

D'autre part, le théorème de Tarski-Seidenberg dit, si $F: \mathbf{R}^{n} \rightarrow \mathbf{R}^{m}$ est une fonction polynomiale et si $V$ est un ensemble semi-algébrique de $\mathbf{R}^{n}$, alors $F(V)$ est un ensemble semi-algébrique de $\mathbf{R}^{m}$ (voir [18]). 
Il est bien connu que le nombre des composantes connexes de telles variétés est fini pour la topologie euclidienne. En effet, si $V$ est un ensemble semi-algébrique défini comme plus haut, alors le nombre de ses composantes connexes est majoré par $(l d+1)(2 l d+1)^{n}$, avec $d=$ $\max \left(\operatorname{deg} P_{i}, \operatorname{deg} Q_{j}\right)$, qui ne dépend pas des coefficients de ces polynômes (voir Proposition 4.4.5 de [4]).

Soit pour $\psi \in \mathfrak{a}^{\star}, \mathcal{A}_{\psi}=G \cdot \Gamma_{f} \cap p^{-1}(\psi)$. Alors il est clair que $\mathcal{M}_{\psi}=G \cdot \mathcal{A}_{\psi}$. Quand le groupe $G$ est nilpotent réel, l'ensemble $\mathcal{A}_{\psi}$ est algébrique et par suite $\mathcal{M}_{\psi}$ est un ensemble semi-algébrique (c'est l'image d'un ensemble algébrique par une fonction polynomiale). Soit $d_{G}$ la dimension maximale des $G$-orbites dans $G \cdot \Gamma_{f}$. On appelle indice de défaut l'entier $i(\psi)=\operatorname{dim} \mathcal{M}_{\psi}-d_{G}$. Alors il est clair que $i(\psi) \geq 0$. C'est cet indice de défaut qui va nous permettre d'écrire une première condition pour la finitude des multiplicités des représentations mixtes. Posons maintenant $d_{H}$ la dimension maximale des $H$-orbites dans $G \cdot \Gamma_{f}$, $d_{A}$ la dimension maximale des $A$-orbites dans $G \cdot \Gamma_{f}$ et finalement $d_{A}^{\prime}$ la dimension maximale des $A$-orbites dans $p\left(G \cdot \Gamma_{f}\right)$. Alors sur un ouvert dense de $\Gamma_{f}$, les dimensions des $G$-orbites, $H$-orbites, $A$-orbites dans $G \cdot \Gamma_{f}$ sont maximales ainsi que la dimension des $A$-orbites dans $p\left(G \cdot \Gamma_{f}\right)$. Nous allons prouver le théorème suivant:

Théorème 4.1. Soit $G$ un groupe de Lie nilpotent, $H$ et $A$ deux sousgroupes fermés connexes de $G$ et $\chi_{f}$ un caractère de $H$. Alors, l'indice de défaut $i(\psi)$ est génériquement nul ou génériquement strictement positif sur $p\left(G \cdot \Gamma_{f}\right)$. De même, les multiplicités de la représentation mixte $\rho(G, H, A, f)$ sont uniformément infinies ou finies et bornées. Ces multiplicités sont finies $\beta$-presque partout si et seulement si pour presque tout $\psi \in p\left(G \cdot \Gamma_{f}\right)$ la triple égalité

$$
\operatorname{dim} \mathcal{M}_{\psi}=2 d_{A}-d_{A}^{\prime}=2 d_{H},
$$

est vérifiée.

Preuve: Rappellons tout d'abord le résultat suivant (voir $[\mathbf{7}]$ ). Soit $\mathcal{Z}=\left\{x \in \mathbf{R}^{m}: Q(x) \neq 0\right\}$ un ouvert de Zariski de $\mathbf{R}^{m}$ et $P$ est une application polynomiale de $\mathbf{R}^{m}$ dans $\mathbf{R}^{d}$. Alors l'ensemble des solutions de l'equation $P(x)=0$ sur $\mathcal{Z}$ est un ensemble fini ou contient une famille à un paramètre de solutions. Dans le cas où il est fini, il est borné par une constante qui ne dépend que de $m, d, \operatorname{deg} P, \operatorname{deg} Q$. Appliquons ce théorème à notre situation: Soit $S$ une section transverse pour toutes les $G$-orbites génériques de $\Gamma_{f}$, obtenue de la manière suivante: Soit $\left(\mathfrak{g}_{i}\right)_{i=0}^{n}$ une suite de Jordan-Hölder de $\mathfrak{g}$ et soit $\left\{Y_{1}, \ldots, Y_{n}\right\}$ une base de JordanHölder extraite de cette suite tel que $Y_{j} \in \mathfrak{g}_{j} \backslash \mathfrak{g}_{j-1}$. Pour $l \in \mathfrak{g}^{*}$, on note 
$\mathfrak{g}(l)$ le radical de la forme bilinéaire $B_{l}$ définie dans la Section 2. Nous notons encore

$$
e(l)=\left\{j \in\{1, \ldots, n\}: Y_{j} \notin \mathfrak{g}_{j-1}+\mathfrak{g}(l)\right\}
$$

et

$$
\Sigma=\left\{e(l): l \in \mathfrak{g}^{*}\right\} .
$$

Pour tout $e \in \Sigma$, on définit la couche

$$
U_{e}=\left\{l \in \mathfrak{g}^{*}: e(l)=e\right\} .
$$

Alors il existe un ordre sur les éléments de $\Sigma=\left\{e^{(1)}<e^{(2)}<\cdots<e^{(\alpha)}\right\}$, tel que $U_{e^{(\alpha)}}$ soit un ouvert de Zariski de $\mathfrak{g}^{*}$ et $U_{e^{(i)}}$ soit un ensemble semi-algébrique pour tout $i \in\{1, \ldots, \alpha\}$. De même, il existe une unique couche $U_{e}$ tel que $U_{e} \cap \Gamma_{f}$ soit un ouvert de Zariski de $\Gamma_{f}$. Ainsi si $e=\left\{j_{1}<\cdots<j_{k}\right\}$, alors $S=U_{e} \cap G \cdot \Gamma_{f} \cap S_{e}$ est une section de croisement pour toutes les $G$-orbites de $\Gamma_{f}$ avec $S_{e}=\operatorname{vect}\left\langle Y_{i}^{*} ; i \notin e\right\rangle$.

Donc toute $G$-orbite générique rencontre $S$ en un point unique. Pour $\psi \in \mathfrak{a}^{\star}, n(\psi)=\#\left[\mathcal{M}_{\psi}^{\prime}\right] / G$ est donné par le nombre des éléments de $S$ qui rencontrent $G \cdot p^{-1}(\psi)$. Le nombre $n(\psi)$ est identiquement infini ou fini et borné comme il est solution de certaines équations polynomiales. Dans le cas où ce nombre est fini, il est majoré par une constante indépendante de $\psi$.

Rappellons que la formule des multiplicités de notre représentation mixte est donnée par

$$
m(\psi)=\sum_{\Omega \in\left[\mathcal{M}_{\psi}\right] / G} n_{\Omega} n_{\Omega}^{\psi} .
$$

D'aprés la formule (12), nous voyons que les nombres $n_{\Omega}^{\psi}$ sont uniformément infinies ou finies et bornées uniformément par rapport à $\psi$ (selon que $d_{G}=2 d_{A}-d_{A}^{\prime}$ ou non). Ainsi les multiplicités de la représentation mixte sont uniformément infinies ou finies et bornées.

Nous montrons finalement que la finitude des multiplicités de $\rho(G, H, A, f)$ est équivalente à $\operatorname{dim} \mathcal{M}_{\psi}=2 d_{A}-d_{A}^{\prime}=2 d_{H}$. Supposons en premier lieu que les multiplicités $m(\psi)$ sont finies presque partout. Il en résulte que génériquement sur $p\left(G \cdot \Gamma_{f}\right)$ les nombres $n(\psi), n_{\Omega}$ et $n_{\Omega}^{\psi}$ sont finis. Donc $\mathcal{M}_{\psi}$ est réunion finie d'orbites, il s'ensuit comme toutes les orbites sont fermées qu'elles sont aussi ouvertes donc elles possèdent la même dimension que la variété $\mathcal{M}_{\psi}$ elle-même. Mais la dimension des orbites génériques est constante et égale à $d_{G}$ d'où $\operatorname{dim} \mathcal{M}_{\psi}=d_{G}$. Ensuite $d_{G}=2 d_{H}$ d'aprés (11) et $d_{G}=2 d_{A}-d_{A}^{\prime}$ d'aprés (12) d'où le 
résultat. Réciproquement, on sait que

$$
d_{G} \leq \operatorname{dim} \mathcal{M}_{\psi}=2 d_{H} \leq d_{G}
$$

alors les $G$-orbites génériques sont ouvertes, donc ouvertes et fermées. Comme elles sont connexes, elles sont les composantes connexes de $\mathcal{M}_{\psi}$. Puisque $\mathcal{M}_{\psi}$ est algébrique, il n'a qu'un nombre fini de composantes connexes, ainsi $n(\psi)$ est fini. De plus l'égalité $d_{G}=2 d_{H}=2 d_{A}-d_{A}^{\prime}$ montre que le nombre $n_{\Omega}$ est fini et $n_{\Omega}^{\psi}$ est aussi fini uniformément par rapport à $\psi$.

Ainsi, nous voyons que la dimension de la variété $\mathcal{M}_{\psi}$ est génériquement constante égale à $d_{G} \operatorname{sur} p\left(G \cdot \Gamma_{f}\right)$ quand $n(\psi)$ est génériquement fini, et donc l'indice de défaut est génériquement nul. Parcontre quand $n(\psi)$ est génériquement infini le raisonnement ci-dessus montre que $i(\psi)$ est génériquement strictement positif.

Corollaire 4.2. Les hypothèses sont celles du théorème précédent. Supposons que $\tau$ est à multiplicités finies et que $\pi_{\mid A}$ est aussi à multiplicités finies, pour presque tout $\pi$ dans le spectre de $\tau$. Alors $\rho(G, H, A, f)$ est à multiplicités finies si et seulement si $i(\psi)=0$ génériquement sur $p\left(G \cdot \Gamma_{f}\right)$.

Remarque 4.3. Dans la preuve du Théorème 4.1, nous remarquons que si \# $\left[\mathcal{M}_{\psi}\right] / G$ est fini, alors toutes les $G$-orbites sont de même dimension. Ainsi $\mathcal{M}^{\prime}{ }_{\psi}=\mathcal{M}_{\psi}$.

Nous étudions maintenant les multiplicités des représentations mixtes dans le cadre des groupes résolubles exponentiels. Nous avons alors la proposition suivante:

Proposition 4.4. Soit $G$ un groupe de Lie résoluble exponentiel, $H$ un sous-groupe fermé connexe de $G$ et $\sigma \in \hat{H}$. Soit a une polarisation $G$-invariante et commune à tous les éléments génériques de $q^{-1}\left(\Omega_{\sigma}^{H}\right)$ et $A=$ expa. Alors, les multiplicités de la représentation mixte $\rho(G, H, A, \sigma)$ coïncident avec celle de $\tau(\sigma)$ et son spectre ne contient que des caractères. Si G est complètement résoluble, alors ces multiplicités sont uniformément bornées si et seulement si l'égalité (11) est satisfaite.

Preuve: Soit $\mathcal{O}_{1}$ l'ouvert dense des éléments de $q^{-1}\left(\Omega_{\sigma}^{H}\right)$ dont la dimension de la $G$-orbite est maximale. Soit $\tilde{\mathcal{O}}_{1}$ l'ensemble des éléments génériques dans $G \cdot q^{-1}\left(\Omega_{\sigma}^{H}\right)$, c'est à dire

$$
\tilde{\mathcal{O}}_{1}=\left\{\phi \in G \cdot q^{-1}\left(\Omega_{\sigma}^{H}\right) / \phi \in G \cdot \phi^{\prime}, \phi^{\prime} \in \mathcal{O}_{1}\right\}=G \cdot \mathcal{O}_{1} .
$$

L'ensemble $\tilde{\mathcal{O}}_{1}$ est un ouvert dans $G \cdot q^{-1}\left(\Omega_{\sigma}^{H}\right)$. Soit $U$ un ouvert non vide de $G \cdot q^{-1}\left(\Omega_{\sigma}^{H}\right)$, alors $G \cdot U$ est un ouvert non vide de $G \cdot q^{-1}\left(\Omega_{\sigma}^{H}\right)$, 
comme

$$
G \cdot\left[G \cdot U \cap q^{-1}\left(\Omega_{\sigma}^{H}\right)\right]=G \cdot U \cap G \cdot q^{-1}\left(\Omega_{\sigma}^{H}\right)=G \cdot U \neq \emptyset,
$$

alors $G \cdot U \cap q^{-1}\left(\Omega_{\sigma}^{H}\right)$ est un ouvert non vide de $q^{-1}\left(\Omega_{\sigma}^{H}\right)$. Ainsi $G$. $U \cap q^{-1}\left(\Omega_{\sigma}^{H}\right) \cap \mathcal{O}_{1} \neq \emptyset$ ce qui veut dire que $G \cdot U \cap \mathcal{O}_{1} \neq \emptyset$ et donc $U \cap G \cdot \mathcal{O}_{1} \neq \emptyset$, et par suite $\tilde{\mathcal{O}}_{1}$ est un ouvert dense dans $G \cdot q^{-1}\left(\Omega_{\sigma}^{H}\right)$.

Posons maintenant $\mathcal{O}=\{p(\phi) ; \operatorname{dim} G \cdot \phi$ est maximale dans $G$. $\left.q^{-1}\left(\Omega_{\sigma}^{H}\right)\right\}=p\left(\tilde{\mathcal{O}}_{1}\right)=p\left(G \cdot \mathcal{O}_{1}\right)$. Par définition de la mesure image

$$
\mu_{G, H, A}^{\sigma}\left(C_{p\left(G \cdot q^{-1}\left(\Omega_{\sigma}^{H}\right)\right) / A}^{\mathcal{O} / A}\right)=(d g \otimes \tilde{\mu})\left(G \times C_{q^{-1}\left(\Omega_{\sigma}^{H}\right)}^{\mathcal{O}_{1}}\right) .
$$

Comme $\mathcal{O}_{1}$ est un ouvert de complémentaire $\tilde{\mu}$-négligeable, alors $\mathcal{O} / A$ est de complémentaire dans $p\left(G \cdot q^{-1}\left(\Omega_{\sigma}^{H}\right)\right) / A, \mu_{G, H, A^{-n e ́ g l i g e a b l e . ~}}^{\sigma}$

Soit $\psi=p(\phi) \in \mathcal{O}$, nous pouvons regarder une extension de $\psi$ à tout $\mathfrak{g}^{\star}$ que nous noterons encore par $\psi$. Vu que $A \cdot \phi=\phi+\operatorname{ad}^{\star} \mathfrak{a} \cdot \phi$, nous voyons que la $A$-orbite est un fermé de $\mathfrak{g}^{\star}$, et donc a est une polarisation de Pukanszky. Ainsi:

$$
A \cdot \phi=\phi+\mathfrak{a}^{\perp}=\psi+\mathfrak{a}^{\perp}=p(\phi)+\mathfrak{a}^{\perp} .
$$

Comme $p^{-1}(\{\psi\})=\psi+\mathfrak{a}^{\perp}$ nous déduisons de la relation (13) que $G \cdot \phi=$ $G \cdot p^{-1}(\{\psi\})$. Ainsi pour tout $\psi=p(\phi) \in \mathcal{O}$, nous avons $G \cdot p^{-1}(\{\psi\})=$ $G \cdot \phi$.

Ainsi $\mathcal{M}_{\psi}(\sigma)=G \cdot \phi$ et $\left.\#\left[G \cdot q^{-1}\left(\Omega_{\sigma}^{H}\right) \cap G \cdot p^{-1}(\{\psi\})\right)\right] / G=1$, ce qui implique que $m_{\sigma}(\psi)=n_{\Omega}^{\sigma} n_{\Omega}^{\psi}$. D'autre part nous savons que $\Omega \cap p^{-1}(A \cdot \psi)=\Omega \cap A \cdot p^{-1}(\{\psi\})$. Il en découle que $\Omega \cap p^{-1}(A \cdot \psi)=$ $\Omega \cap(A \cdot \phi)=A \cdot \phi$. Finalement, $n_{\Omega}^{\psi}=\#\left[\left(\Omega \cap p^{-1}(A . \psi)\right)\right] / A=1$. D'où $m_{\sigma}(\psi)=n_{\Omega}$. D'autre part, pour $\psi \in \mathcal{O}$, nous avons $\psi([\mathfrak{a}, \mathfrak{a}])=0$ et donc le spectre de $\rho(G, H, A, \sigma)$ ne fait intervenir que des caractères. Quand $G$ est complètement résoluble, les multiplicités de $\tau(\sigma)$ sont uniformément bornées si (11) est vérifiée, sinon elles sont uniformément infinies.

Corollaire 4.5. Supposons que $G$ est nilpotent et que $\tau(f)$ est à multiplicités finies presque partout. Supposons que $\operatorname{Ad}_{G}([\mathfrak{a}, \mathfrak{a}]) \subset \mathfrak{h} \cap \operatorname{ker} f$ et que $\operatorname{dim}(\mathfrak{g} / \mathfrak{a})=\frac{1}{2} d_{G}$. Alors les multiplicités de $\rho(G, H, A, f)$ sont finies presque partout.

La première condition est trivialement vérifiée quand $\mathfrak{a}$ est abélienne. Ainsi on retrouve le résultat du Théorème $3.1 \mathrm{du}[\mathbf{1 2}]$. 
Preuve: Soit $\phi$ un élément générique dans $\Gamma_{f}$, alors $\phi=f+\phi_{1}$ avec $\phi_{1} \in \mathfrak{h}^{\perp}$ et $g \cdot \phi([\mathfrak{a}, \mathfrak{a}])=0$ pour tout $g \in G$. D'autre part:

$$
\begin{aligned}
\operatorname{dim} \mathfrak{a} & =\operatorname{dim} \mathfrak{g}-\frac{1}{2} d_{G} \\
& =\operatorname{dim} \mathfrak{g}-\frac{1}{2}(\operatorname{dim} \mathfrak{g}-\operatorname{dim} \mathfrak{g}(\phi)) \\
& =\frac{1}{2}(\operatorname{dim} \mathfrak{g}+\operatorname{dim} \mathfrak{g}(\phi)) .
\end{aligned}
$$

Ainsi a est une polarisation réelle en tout élément de l'orbite $G \cdot \phi \cdot \mathrm{Vu}$ que $\mathfrak{g}$ est nilpotent alors $\mathfrak{a}$ est une polarisation de Pukanszky. Ainsi le reste de la preuve découle de la fin de la démonstration de la proposition précédente sans utiliser le fait que $\mathfrak{a}$ est un idéal de $\mathfrak{g}$. On obtient que les multiplicités de la représentation $\rho(G, H, A, f)$ coincident avec celles de la représentation $\tau(f)$ qui sont finies presque partout par hypothèse.

L'exemple suivant montre que les conditions du Corollaire 4.5 sont suffisantes et non necessaires pour assurer la finitude des multiplicités de la représentation $\rho(G, H, A, f)$.

Exemple 4.6. Soit $G$ le groupe de Lie nilpotent, connexe et simplement connexe, d'algèbre de Lie $\mathfrak{g}$ engendrée par $\left(e_{1}, \ldots, e_{6}\right)$ et dont les crochets non nuls sont donnés par:

$$
\begin{aligned}
& {\left[e_{6}, e_{5}\right]=-e_{4},} \\
& {\left[e_{6}, e_{4}\right]=-e_{3},} \\
& {\left[e_{6}, e_{3}\right]=-e_{2},} \\
& {\left[e_{5}, e_{2}\right]=-e_{1},} \\
& {\left[e_{4}, e_{3}\right]=e_{1} .}
\end{aligned}
$$

Posons $\mathfrak{h}=\operatorname{vect}\left(e_{2}, e_{3}, e_{6}\right)$ et $\mathfrak{a}=\operatorname{vect}\left(e_{1}, e_{2}, e_{3}, e_{6}\right)$. Alors $\mathfrak{h}$ et $\mathfrak{a}$ sont deux sous-algèbres de $\mathfrak{g}$. Notons $f=\lambda e_{3}^{\star}$. Les orbites génériques dans $\Gamma_{\tau}$ sont de dimension $d_{G}=4$. En effet, un simple calcul montre que pour $x \neq 0$, l'orbite $G \cdot f_{x}$ de $f_{x}=\lambda e_{3}^{\star}+x e_{1}^{\star}$ est décrite comme:

$\Omega_{x}=\left\{x e_{1}^{\star}+t_{2} e_{2}^{\star}+t_{3} e_{3}^{\star}+t_{4} e_{4}^{\star}+t_{5} e_{5}^{\star}+\frac{1}{2 x}\left(t_{3}^{2}-2 t_{2} t_{4}-\lambda^{2}\right) e_{6}^{\star} ; t_{i} \in \mathbf{R}\right\}$.

Ainsi, $\tau \simeq \int_{\mathbf{R}}^{\oplus} \pi_{x} d x$ où $\pi_{x}$ est la représentation associée à l'orbite $\Omega_{x}$. L'hypothèse $n_{\Omega}$ est fini, pour tout $\Omega$ générique dans $G \cdot \Gamma_{\tau}$ est vérifiée. 
D'autre part, nous avons:

$$
\pi_{x \mid A} \simeq \int_{\mathbf{R}}^{\oplus} \rho_{x, y} d y
$$

où $\rho_{x, y}$ est la représentation dans $\hat{A}$ associée à l'orbite $A \cdot\left(x e_{1}^{\star}+y e_{2}^{\star}\right)$. D'où

$$
\tau_{\mid A} \simeq \int_{\mathbf{R}^{2}}^{\oplus} \pi_{x, y}^{\circ} d x d y
$$

D'autre part le sous-groupe $A$ est de codimension $2=\frac{1}{2} d_{G}$, mais l'hypothèse $\operatorname{Ad}_{G}([\mathfrak{a}, \mathfrak{a}]) \subset \mathfrak{h} \cap \operatorname{ker} f$ du Corollaire 4.5 n'est pas vérifiée alors que $m(\psi)=1$ pour tout $\psi$ générique dans $p\left(G \cdot \Gamma_{\tau}\right)$.

\section{Cas des sous-groupes invariants}

Dans toute cette section, nous supposons que $G$ est un groupe de Lie résoluble exponentiel; $H$ et $A$ sont deux sous-groupes fermés connexes, normaux de $G$. Pour $\psi \in \mathfrak{a}^{*}$, soit $\mathcal{B}_{\psi}=\Gamma_{f} \cap G \cdot p^{-1}(\psi)$, alors il est clair que $\mathcal{B}_{\psi}$ est un ensemble analytique (i.e. ensemble des zéros communs d'une famille finie de fonctions analytiques) et que $\mathcal{M}_{\psi}=G \cdot \mathcal{B}_{\psi}$.

Comme dans la section précédente on pose $d_{H}$ la dimension maximale des $H$-orbites dans $G \cdot \Gamma_{f}$ et $d_{A}$ la dimension maximale des $A$-orbites dans $G \cdot \Gamma_{f}$ et soit $d_{A}^{\prime}$ la dimension maximale des $A$-orbites dans $p\left(G \cdot \Gamma_{f}\right)$. Conformément au cas nilpotent, génériquement sur $\Gamma_{f}$, les dimensions des $G$-orbites, $H$-orbites, $A$-orbites dans $G \cdot \Gamma_{f}$ sont maximales ainsi que la dimension des $A$-orbites dans $p\left(G \cdot \Gamma_{f}\right)$.

Soient $\phi \in \Gamma_{f}, g \in G$ et $\psi=p(g \cdot \phi) \in p\left(G \cdot \Gamma_{f}\right)$. Notons

$$
\mathfrak{k}=(\mathfrak{a} \cap \mathfrak{h})^{\phi}=(\mathfrak{a} \cap \mathfrak{h})^{f}=\{X \in \mathfrak{g}: f[X, \mathfrak{a} \cap \mathfrak{h}]=0\},
$$

et

$$
K=\exp (\mathfrak{k})=\left\{g \in G: g \cdot \phi_{\mid \mathfrak{a} \cap \mathfrak{h}}=\phi_{\mid \mathfrak{a} \cap \mathfrak{h}}\right\} .
$$

Soient les sous-espaces $\mathfrak{w}$ de $\mathfrak{g}, \mathfrak{u}$ de $\mathfrak{a}$ et $\mathfrak{v}$ de $\mathfrak{h}$ telle que

$$
\mathfrak{g}=\mathfrak{w} \oplus(\mathfrak{a}+\mathfrak{h}), \mathfrak{h}=\mathfrak{v} \oplus(\mathfrak{a} \cap \mathfrak{h}) \text {, et } \mathfrak{a}=\mathfrak{u} \oplus(\mathfrak{a} \cap \mathfrak{h}) .
$$

Nous allons commencer par prouver le lemme suivant:

\section{Lemme 5.1.}

$$
\mathcal{B}_{\psi}=\tilde{\phi}+p(K \cdot \phi)+(\mathfrak{a}+\mathfrak{h})^{\perp},
$$

où $\tilde{\phi}$ est la forme linéaire sur $\mathfrak{g}$ définie par $\tilde{\phi}=f$ sur $\mathfrak{v}$ et $\tilde{\phi}=0$ ailleurs. En particulier, les ensembles $\mathcal{B}_{\psi}$ et $\mathcal{M}_{\psi}$ sont connexes. 
Preuve: Soit $\psi=p(g \cdot \phi) \in p\left(G \cdot \Gamma_{f}\right)$ pour un certain $g \in G$ et $\phi \in \Gamma_{f}$. Remarquons tout d'abord que $\mathcal{B}_{\psi}=\left(\phi+\mathfrak{h}^{\perp}\right) \cap G \cdot\left(\phi+\mathfrak{a}^{\perp}\right) \neq \emptyset$. Un élément $l$ de $\mathfrak{g}^{*}$ est dans $\mathcal{B}_{\psi}$ si et seulement si $l$ s'écrit sous la forme $l=$ $\phi+\alpha=k \cdot \phi+\beta$ pour certaines formes linéaires $\alpha \in \mathfrak{h}^{\perp}, \beta \in \mathfrak{a}^{\perp}$ et un élément $k \in G$. Comme $k \cdot \phi=\phi$ sur $\mathfrak{h} \cap \mathfrak{a}$, alors $k \in K$. Ainsi $\alpha=k \cdot \phi-\phi$ sur $\mathfrak{a}$ et $\beta=\phi-k \cdot \phi$ sur $\mathfrak{h}$. Posons $F$ l'application: $K \times(\mathfrak{a}+\mathfrak{h})^{\perp} \rightarrow \mathfrak{g}^{*}$ définie $\operatorname{par}(k, \varphi) \mapsto k \cdot \phi+\beta(k)+\varphi$, avec $\beta(k) \in \mathfrak{a}^{\perp}$ et est définie par $\beta(k)=f-k \cdot f$ sur $\mathfrak{v}$ et $\beta(k)=0$ sur $\mathfrak{w}$. alors $\mathcal{B}_{\psi}$ est contenu dans l'image de $K \times(\mathfrak{a}+\mathfrak{h})^{\perp}$ par l'application $F$. Inversement soit $l$ un élément de cette image; $l=k \cdot \phi+\beta(k)+\varphi$. Alors on peut écrire $l$ sous la forme $l=f+\alpha$ avec $\alpha \in \mathfrak{h}^{\perp}$ définit par $\alpha=k \cdot \phi$ sur $\mathfrak{u}$ et $\alpha=\varphi+k \cdot \phi-f$ sur $\mathfrak{w}$; et donc $l$ est un élément de $f+\mathfrak{h}^{\perp}$. De même, nous voyons que $l=k \cdot \phi+\gamma$ avec $\gamma \in \mathfrak{a}^{\perp}$ définit par $\gamma=\beta(k)$ sur $\mathfrak{v}$ et $\gamma=\varphi$ sur $\mathfrak{w}$ et donc $l$ est un élément de $G \cdot \phi+\mathfrak{a}^{\perp}$. Ceci nous conduit au fait que $\mathcal{B}_{\psi}$ est l'image de $K \times(\mathfrak{a}+\mathfrak{h})^{\perp}$ par $F$.

Ainsi on peut voir que $\mathcal{B}_{\psi}=\tilde{\phi}+p(K \cdot \phi)+(\mathfrak{a}+\mathfrak{h})^{\perp}$, tout en considérant les éléments de $p(K \cdot \phi)$ comme des éléments de $\mathfrak{g}^{*}$ par extension triviale.

Nous sommes prêts maintenant à prouver le résultat principal de cette section.

Théorème 5.2. Soient $G$ un groupe de Lie résoluble exponentiel; $H$ et $A$ deux sous-groupes fermés connexes, normaux de $G$ et $\chi_{f}$ un caractère unitaire de $H$. Alors les multiplicités de la représentation mixte $\rho(G, H, A, f)$ sont uniformément infinies ou uniformément égales à un. Une condition nécessaire et suffisante pour que $\rho(G, H, A, f)$ soit sans multiplicités est que pour presque tout $\psi \in p\left(G \cdot \Gamma_{f}\right)$ nous ayons $\operatorname{dim} \mathcal{B}_{\psi}=$ $d_{H}=d_{A}-\frac{1}{2} d_{A}^{\prime}$.

Preuve: Conformément à la formule (7), nous voyons que $m(\psi)=1$ ou $+\infty$. En effet, si $m(\psi)<\infty$, alors $\#\left[\mathcal{M}^{\prime}{ }_{\psi}\right] / G<\infty$ et pour toute $\Omega$ générique dans $G \cdot \Gamma_{f}, n_{\Omega}=n_{\Omega}^{\psi}=1$. Posons donc $\mathcal{M}_{\psi}^{\prime}=\bigcup_{i=1}^{m} G \cdot \phi_{i}$, où les $G \cdot \phi_{i}$ sont des orbites génériques. Ce qui donne que $\mathcal{B}^{\prime}{ }_{\psi}=$ $\bigcup_{i=1}^{m} G \cdot \phi_{i} \cap \Gamma_{f}=\bigcup_{i=1}^{m} H \cdot \phi_{i}=\mathcal{B}_{\psi}$ car pour toute forme linéaire $\phi \in \Gamma_{f}$, $\operatorname{dim} H \cdot \phi=\operatorname{dim} H \cdot f$. Nous avons $H \cdot \phi_{i}=\phi_{i}+\operatorname{ad}^{\star} \mathfrak{h} \cdot \phi_{i}=\phi_{i}+\operatorname{ad}^{\star} \mathfrak{h} \cdot f$, par suite $H \cdot \phi_{i}$ est un fermé et ouvert de $\mathcal{B}_{\psi}$ qui est connexe. Ainsi $m=1$ et $\mathcal{B}_{\psi}=H \cdot \phi$ pour un certain $\phi$ dans $\Gamma_{f}$, ce qui donne que 
$\mathcal{M}_{\psi}=G \cdot \phi$ et $m(\psi)=1$. Prouvons maintenant que:

(14) $m(\psi)=1 \Longleftrightarrow \operatorname{dim} \mathcal{B}_{\psi}=\operatorname{dim} H \cdot \phi=\operatorname{dim} A \cdot \phi-\frac{1}{2} \operatorname{dim} A \cdot \psi$.

En effet, supposons d'abord que $m(\psi)=1$. Dans ce cas, nous avons $\mathcal{M}_{\psi}=\mathcal{M}_{\psi}^{\prime}=G \cdot \phi, n_{G \cdot \phi}=1$ et $n_{G \cdot \phi}^{\psi}=1$. Ainsi, $\mathcal{B}_{\psi}=H \cdot \phi$ et donc $\operatorname{dim} \mathcal{B}_{\psi}=\operatorname{dim} H \cdot \phi=\operatorname{dim} A \cdot \phi-\frac{1}{2} \operatorname{dim} A \cdot p(\phi)$. Réciproquement, si $\operatorname{dim} \mathcal{B}_{\psi}=\operatorname{dim} H \cdot \phi$, alors $H \cdot \phi$ est un ouvert de la varieté $\mathcal{B}_{\psi}$, qui est connexe. Vu que $H \cdot \phi$ est un fermé, nous avons $\mathcal{B}_{\psi}=H \cdot \phi$. Aussi $\mathcal{M}_{\psi}=G \cdot \phi$ et $\mathcal{B}_{\psi}=\mathcal{M}_{\psi} \cap \Gamma_{f}=\Gamma_{f} \cap G \cdot \phi=H \cdot \phi$. Ceci nous conduit au fait que $n_{G \cdot \phi}=1$, ainsi $\operatorname{dim} G \cdot \phi=2 H \cdot \phi$ et le fait que $\operatorname{dim} H \cdot \phi=$ $\operatorname{dim} A \cdot \phi-\frac{1}{2} \operatorname{dim} A \cdot \psi$ nous permet de déduire que $n_{G \cdot \phi}^{\psi}=1$. Ceci termine la preuve de (14). Maintenant, remarquons que $\operatorname{dim} \mathcal{B}_{\psi}=\operatorname{dim} p(K \cdot \phi)+$ $\operatorname{dim}(\mathfrak{a}+\mathfrak{h})^{\perp}=\operatorname{dim} K \cdot p(\phi)+\operatorname{dim}(\mathfrak{a}+\mathfrak{h})^{\perp}$ qui est génériquement constant sur $\Gamma_{f}$. Ainsi les multiplicités de $\rho(G, H, A, f)$ sont uniformément un ou l'infinie.

Corollaire 5.3 (Alignement au cas nilpotent). Les hypothèses sont celles du théorème précédent. Alors la représentation $\rho(G, H, A, f)$ est sans multiplicités si et seulement si pour presque tout $\psi \in p\left(G \cdot \Gamma_{f}\right)$, $\mathcal{M}_{\psi}$ est une variété dont la dimension vérifie

$$
\operatorname{dim} \mathcal{M}_{\psi}=2 d_{H}=2 d_{A}-d_{A}^{\prime} .
$$

Preuve: Supposons que $\mathcal{M}_{\psi}$ est une variété dont la dimension vérifie $\operatorname{dim} \mathcal{M}_{\psi}=2 d_{H}=2 d_{A}-d_{A}^{\prime}$. Posons $\mathcal{M}_{\psi}=\cup_{i \in I} G \cdot \phi_{i}$. Alors pour tout $i \in I ; \operatorname{dim} G \cdot \phi_{i} \leq \operatorname{dim} \mathcal{M}_{\psi}=2 \operatorname{dim} H \cdot \phi_{i} \leq \operatorname{dim} G \cdot \phi_{i}$. Ainsi $\operatorname{dim} \mathcal{M}_{\psi}=$ $\operatorname{dim} G \cdot \phi_{i}$ et donc toute orbite est un ouvert et par suite un fermé de $\mathcal{M}_{\psi}$ qui est connexe, on en déduit que $I$ a un seul élément et que $\mathcal{M}_{\psi}=G \cdot \phi$ et donc $\operatorname{dim} G \cdot \phi=2 \operatorname{dim} H \cdot \phi$. Finalement $\mathcal{B}_{\psi}=G \cdot \phi \cap \Gamma_{f}=H \cdot \phi$, d'où $\operatorname{dim} \mathcal{B}_{\psi}=\operatorname{dim} H \cdot \phi$. L'hypothèse $\operatorname{dim} H \cdot \phi=d_{A}-\frac{1}{2} d_{A}^{\prime}$ et le Théorème 5.2 nous permettent de déduire que la représentation $\rho(G, H, A, f)$ est sans multiplicités.

Réciproquement si les multiplicités de $\rho(G, H, A, f)$ sont uniformément finies, alors pour presque tout $\psi \in p\left(G \cdot \Gamma_{f}\right), m(\psi)=1$. Dans ce cas $\mathcal{M}_{\psi}=G \cdot \phi$ qui est une variété avec $\phi$ est un élément générique dans $\Gamma_{f}$ vérifiant $\psi=p(g \cdot \phi)$. Aussi $n_{G \cdot \phi}^{\psi}=n_{G \cdot \phi}=1$ et $\operatorname{donc} \operatorname{dim} \mathcal{M}_{\psi}=$ $\operatorname{dim} G \cdot \phi=2 d_{H}=2 d_{A}-d_{A}^{\prime}$.

Corollaire 5.4. Soient $G$ un groupe de Lie résoluble exponentiel; $H$ et $A$ deux sous-groupes fermés connexes, normaux de $G$ et $\chi_{f}$ un caractère unitaire de $H$. Supposons que $[\mathfrak{a}, \mathfrak{a}] \subset \mathfrak{h} \cap \operatorname{ker} f$ et $\operatorname{dim} \mathfrak{g} / \mathfrak{a}=\frac{1}{2} d_{G}$. Si $\tau$ est à multiplicités finies, alors $\rho(G, H, A, f)$ est sans multiplicités. 
Preuve: Les hypothèses nous conduisent au fait que $\mathfrak{a}$ est une polarisation commune à tous les éléments dont la dimension de la $G$-orbite est maximale de $\Gamma_{f}$. Les Théorèmes 3.6 et 5.2 , nous permettent de conclure.

Nous allons maintenant étudier le cas particulier où $A=H$.

Proposition 5.5. Soit $G$ un groupe de Lie résoluble exponentiel, $H$ un sous-groupe fermé connexe, normal de $G$ et $\chi_{f}$ un caractère de $H$. Alors:

$$
\rho(G, H, f) \simeq m(f, \mathfrak{h}) \int_{p(G \cdot f)}^{\oplus} \chi_{\psi} d \mu_{G, H}^{\pi_{f}}(\psi) \simeq m(f, \mathfrak{h}) \pi_{f_{\mid H}},
$$

où $m(f, \mathfrak{h})=1$ ou l'infini selon que $\mathfrak{h} \in M(f, \mathfrak{g})$ ou non.

Preuve: Soit

$$
K=H^{f}=\left\{g \in G: g \cdot\left(f_{\mid \mathfrak{h}}\right)=f_{\mid \mathfrak{h}}\right\} .
$$

L'espace de la représentation $\rho=\rho(G, H, f)$ est $L^{2}(G / H, f)$. Pour $\xi \in$ $L^{2}(G / H, f)$, nous avons

$$
\rho(h) \xi(g)=\chi_{g \cdot f}(h) \xi(g) \quad \forall h \in H, \quad \forall g \in G,
$$

et

$$
L^{2}(G / H, f)=L^{2}(G / K) \otimes L^{2}(K / H, f) \simeq \int_{\Omega}^{\oplus} L^{2}(K / H, \varphi) d \mu_{G, H}^{f}(\varphi),
$$

où $\Omega=G \cdot f_{\mid \mathfrak{h}}=G / K$. Ainsi, si $H=K$, alors $H$ est une polarisation de Pukanszky en $f$ et $\rho \simeq \int_{\Omega}^{\oplus} \chi_{\varphi} d \mu_{G, H}^{f}(\varphi) \simeq \pi_{f_{\mid H}}$. Si $H \neq K$, alors $\rho \simeq \int_{\Omega}^{\oplus} \infty \chi_{\varphi} d \mu_{G, H}^{f}(\varphi) \simeq \infty \pi_{f \mid H}$.

Exemples 5.6. Soit $G$ un groupe de Lie résoluble exponentiel d'algèbre de Lie $\mathfrak{g}$. On sait que $\left(\operatorname{Ind}_{\{e\}}^{G} 1\right)$ est la représentation régulière gauche $\lambda_{G}$ qui se réalise sur $L^{2}(G)$ par $\lambda_{G}(g) \xi(x)=\xi\left(g^{-1} x\right)$ pour tout $g, x \in G$ et $\xi \in L^{2}(G)$. Ici $f=0$ et $H=\{e\}$. Donc $\mathfrak{h}$ n'est pas une polarisation en $f$, et $\rho(G,\{e\}, 0)=\lambda_{\left.G\right|_{\{e\}}} \simeq \infty \cdot 1$.

Prenons maintenant $H$ un sous-groupe normal de codimension 1 dans $G$ tel que $\mathfrak{h}$ soit subordonnée à $f$ et $p: \mathfrak{g}^{\star} \rightarrow \mathfrak{h}^{\star}$ la projection canonique. Notons $\mathfrak{g}=\mathfrak{h} \oplus \mathbf{R} X$. Si $\mathfrak{g}(f) \subseteq \mathfrak{h}$, alors $\mathfrak{h}$ est une polarisation en $f$ et on a $\rho(G, H, f) \simeq \int_{\mathbf{R}}^{\oplus} \chi_{p(\exp t X \cdot f)} d t$. Si $\mathfrak{g}(f) \not \subset \mathfrak{h}$, dans ce cas $\mathfrak{h}$ n'est pas une polarisation en $f$ et on a $\rho(G, H, f) \simeq \infty \chi_{p(f)}$.

Soit enfin $G$ le groupe de Boidol, son algèbre de Lie $\mathfrak{g}$ est engendrée par les vecteurs $A, X, Y, Z$ tels que $[A, X]=X ;[A, Y]=-Y ;[X, Y]=Z$. Prenons $f_{\alpha}=\alpha Z^{\star} ; \alpha \neq 0$. Si $H=\exp (\mathbf{R} Z)$, alors $\mathfrak{h}$ n'est pas une polarisation en $f_{\alpha}$, ainsi $m(f, \mathfrak{h})=+\infty$ et $\rho\left(G, H, f_{\alpha}\right) \simeq \infty \chi_{\alpha Z^{\star}}$. 
Si $H=\exp (\mathbf{R} Z \oplus \mathbf{R} X)$ alors $\mathfrak{h}$ n'est pas une polarisation en $f$ aussi car $\mathfrak{h}$ n'est pas maximale et $m(f, \mathfrak{h})=+\infty$. On obtient $\rho\left(G, H, f_{\alpha}\right) \simeq$ $\int_{\mathbf{R}}^{\oplus} \infty \chi_{\left(\alpha Z^{\star}+\beta X^{\star}\right)} d \beta$.

Remerciements. Nous remercions vivement le Professeur R. L. Lipsman pour nous avoir envoyer ses articles sur le sujet. Nous remercions aussi le referee pour les modifications qu'il les a suggérées et pour avoir proposer des preuves plus simples pour le Lemme 5.1 et la Proposition 5.5.

\section{Références}

[1] A. Baklouti, Nouvelle désintégration lisse de $L^{2}(G)$ pour les groupes résolubles exponentiels, J. Lie Theory 8(1) (1998), 25-50.

[2] A. Baklouti et J. Ludwig, Désintégration des représentations monomiales des groupes de Lie nilpotents, J. Lie Theory 9(1) (1999), 157-191.

[3] A. Baklouti et J. Ludwig, Entrelacement des restrictions des représentations unitaires des groupes de Lie nilpotents, Ann. Inst. Fourier (Grenoble) 51(2) (2001), 395-429.

[4] R. Benedetti et J.-J. Risler, "Real algebraic and semi-algebraic sets. Actualités Mathématiques", Hermann, Paris, 1990.

[5] P. Bernat, N. Conze, M. Duflo, M. Lévy-Nahas, M. Raïs, P. Renouard et M. Vergne, "Représentations des groupes de Lie résolubles", Monographies de la Société Mathématique de France 4, Dunod, Paris, 1972.

[6] J. Bochnak, M. Coste et M.-F. Roy, "Géométrie algébrique réelle", Ergebnisse der Mathematik und ihrer Grenzgebiete (3) 12, Springer-Verlag, Berlin, 1987.

[7] L. Corwin, F. P. Greenleaf et G. Grélaud, Direct integral decompositions and multiplicities for induced representations of nilpotent Lie groups, Trans. Amer. Math. Soc. 304(2) (1987), 549-583.

[8] H. Fujiwara, Représentations monomiales des groupes de Lie résolubles exponentiels, in: "The orbit method in representation theory" (Copenhagen, 1988), Progr. Math. 82, Birkhäuser Boston, Boston, MA, 1990, pp. 61-84.

[9] H. Fujiwara, Sur les restrictions des représentations unitaires des groupes de Lie résolubles exponentiels, Invent. Math. 104(3) (1991), 647-654.

[10] H. Fujiwara, Représentations monomiales des groupes de Lie nilpotents, Pacific J. Math. 127(2) (1987), 329-352. 
[11] A. A. Kirillov, Unitary representations of nilpotent Lie groups, (Russian), Uspehi Mat. Nauk 17(4) (1962), 57-110.

[12] R. L. LiPSMAN, The up-down formula for nil-homogeneous spaces, Ann. Mat. Pura Appl. (4) 166 (1994), 291-300.

[13] R. L. LiPSMAn, Restricting representations of completely solvable Lie groups, Canad. J. Math. 42(5) (1990), 790-824.

[14] R. L. Lipsman, Induced representations of completely solvable Lie groups, Ann. Scuola Norm. Sup. Pisa Cl. Sci. (4) 17(1) (1990), $127-164$.

[15] R. L. Lipsman, The multiplicity function on exponential and completely solvable homogeneous spaces, Geom. Dedicata 39(2) (1991), $155-161$.

[16] J. Ludwig, Dual topology of diamond groups, J. Reine angew. Math. 467 (1995), 67-87.

[17] G. W. MACKEY, "The theory of unitary group representations", Chicago Lectures in Mathematics, University of Chicago Press, Chicago, Ill.-London, 1976.

[18] A. TARSKI, "A decision method for elementary algebra and geometry", 2nd ed., University of California Press, Berkeley and Los Angeles, Calif., 1951.

Département de Mathématiques

Faculté des Sciences de Sfax

Route de Soukra

3038 Sfax

Tunisie

E-mail address: Ali.Baklouti@fss.rnu.tn

E-mail address: Amira.Ghorbel@fss.rnu.tn

E-mail address: Hatem.Hamrouni@ipeigb.rnu.tn 